\title{
Dynamic Walking on Compliant and Uneven Terrain using DCM and Passivity-based Whole-body Control
}

\author{
George Mesesan, Johannes Englsberger, Gianluca Garofalo, Christian Ott, Alin Albu-Schäffer
}

\begin{abstract}
This paper presents a complete trajectory generation and control approach for achieving a robust dynamic walking gait for humanoid robots over compliant and uneven terrain. The work uses the concept of Divergent Component of Motion (DCM) for generating the center of mass (CoM) trajectory, and Cartesian polynomial trajectories for the feet. These reference trajectories are tracked by a passivity-based whole-body controller, which computes the joint torques for commanding our torque-controlled humanoid robot TORO. We provide the implementation details regarding the trajectory generation and control that help preventing discontinuities in the commanded joint torques, which facilitates precise trajectory tracking and robust locomotion. We present extensive experimental results of TORO walking over rough terrain, grass, and, to the best of our knowledge, the first report of a humanoid robot walking over a soft gym mattress.
\end{abstract}

\section{INTRODUCTION}

Locomotion of legged robots is a challenging problem due to its hybrid dynamics (discrete contact sequencing and continuous whole-body motion), the constraints on the contact forces, and the high dimensionality of the system dynamics. A widely used approach for online gait generation is to focus on the center of mass (CoM) dynamics, which covers the most important effects for legged locomotion. This approach has been shown to be consistent with legged locomotion of biological systems [1], and was successfully applied in legged robotics to both walking [2], [3] and running [4], [5]. One of the most popular models for robotic walking control is the Linear Inverted Pendulum (LIP) [6]. More recently, the concept of Divergent Component of Motion (DCM) [7], [8] (also known as instantaneous Capture Point (iCP) [9]) was introduced with the goal of simplifying the trajectory generation and control by ignoring the stable component of the CoM dynamics. In our previous work [10], [11], we presented an efficient algorithm based on DCM for creating continuous multi-step CoM trajectories via piecewise interpolation over a sequence of waypoints.

Early humanoid robots could only be position-controlled, which facilitates the precise tracking of the foot trajectory during walking. At the same time, admittance control using feedback from force torque sensors in the feet provides sufficient compliance to enable walking on uneven terrain [12], [13]. Recently, several torque-controllable robots have been developed [14]-[16]. These have several advantages over position-controlled ones: First, torque control provides a higher bandwidth regarding contact force modulation

All authors are with the Institute of Robotics and Mechatronics, German Aerospace Center (DLR), Wessling, Germany. Contact: george.mesesan@dlr.de
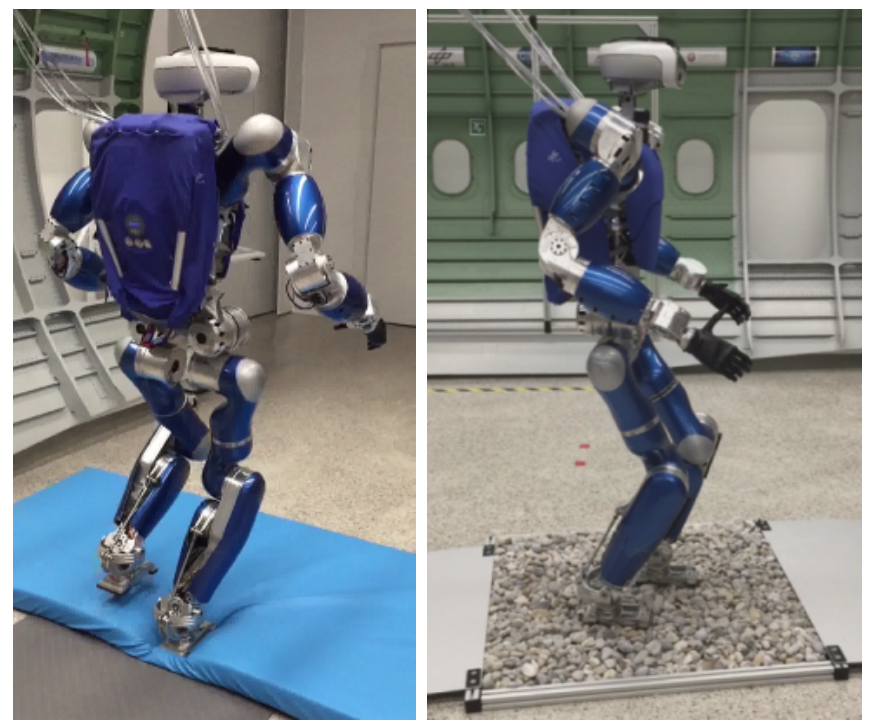

Fig. 1: TORO walking on compliant (left) and on rough terrain (right).

compared to admittance control, such that more compliant interaction with the ground can be realized. Second, torque controlled robots enable the interaction with the environment at arbitrary contact points [17], which is an important feature in the context of multi-contact locomotion. Finally, the compliance of the torque-controlled robots makes them more suitable for applications in the field of human-robot interaction, particularly when considering clamping safety.

As torque-controllable robots become available and more diverse, several controllers have been presented in the literature. The approach taken by Feng et al. [18] combines inverse kinematics and inverse dynamics, while Hopkins et al. [19] use a "leaky integrator" for generating joint velocity references from accelerations computed by a QP solver, with the goal of overcoming the imperfections of the robotic hardware. Stephens et al. [20] present pushrecovery experiments based on joint tracking using a low gain impedance combined with feed-forward torques. A passivity-based whole-body torque controller for balancing in multi-contact scenarios has been proposed by Henze et al. in [21], and a walking controller based on DCM control and inverse dynamics has been demonstrated in experiments with the humanoid robot TORO in our previous work [22].

The main contributions of this work are: (i) we generalize the passivity-based whole-body controller introduced in [21] from a purely Cartesian formulation to a generalized tasks 
definition, including DCM tracking control, Cartesian endeffector and joint level subtasks; (ii) within this framework, we present a gait generation and control method for dynamic walking with a strong focus on avoiding discontinuities in the commanded torques, which we found to be an important factor in achieving robust dynamic walking; (iii) we introduce a stabilization method for the feet in contact that improves the overall controller performance when the rigid contact assumption (zero translational and rotational velocity) does not hold, such as is often the case when walking over compliant or uneven terrain.

The rest of the paper is organized as follows: Section II describes the trajectory generation approach designed such that discontinuities in the commanded torques are avoided. In Section III, we present the passivity-based whole-body controller using generalized tasks, followed in Section IV by implementation details for dynamic walking including a stance foot stabilization method. Section V describes the robotic hardware, the software implementation, and the performed experiments that demonstrate the robustness of the overall approach. Section VI concludes the paper.

\section{REFERENCE TRAJECTORY GENERATION}

Our main goal for trajectory generation is to avoid discontinuities in the reference trajectories, which would affect the commanded torques through both feed-forward and feedback terms. Discontinuities in the commanded joint torques are undesirable for two reasons: First, a real robot has limited torque bandwidth. This means that the motor torques cannot follow the commanded joint torques in case of discontinuities, which leads to discrepancies between command and action. Second, torque discontinuities tend to excite unmodeled joint and link elasticities, often leading to vibrations such as reported in [22].

\section{A. Continuous CoM reference trajectory}

The CoM trajectory is generated using the concept of the three-dimensional Divergent Component of Motion (DCM), which was defined in [7] as a linear combination of the CoM position $\boldsymbol{x}_{c}$ and velocity $\dot{\boldsymbol{x}}_{c}$ :

$$
\boldsymbol{\xi}=\boldsymbol{x}_{c}+b \dot{\boldsymbol{x}}_{c},
$$

where $b$ is a time constant defined as $b=\sqrt{\frac{\Delta z}{g}}$. Here, $\Delta z$ represents the average CoM height above the ground surface, and $g$ denotes the gravitational constant. A closely related concept, the Virtual Repellent Point (VRP), was introduced in [7] as a linear combination of the CoM position $\boldsymbol{x}_{c}$ and acceleration $\ddot{\boldsymbol{x}}_{c}$ :

$$
\boldsymbol{v}=\boldsymbol{x}_{c}-b^{2} \ddot{\boldsymbol{x}}_{c}
$$

The VRP encodes the effects of the total force $\boldsymbol{f}_{c}$ (gravity and all external forces) acting on the CoM, as the CoM acceleration is proportional to the total force $\boldsymbol{f}_{c}=m \ddot{\boldsymbol{x}}_{c}$ (Newton's 2nd law). From (1) and (2) we find the relation between DCM and VRP to be

$$
\boldsymbol{v}=\boldsymbol{\xi}-b \dot{\boldsymbol{\xi}} .
$$

As shown in our previous work [11], a multi-step preview trajectory for the CoM can be efficiently computed as a closed-form, $C^{2}$ continuous trajectory by starting with a piecewise linear interpolation of the VRP trajectory over a sequence of waypoints, and solving the differential equations (1) and (3).

For walking, the VRP waypoints are placed over the foot centers at a height equal to $\Delta z$. Then, the VRP trajectory is generated by keeping the VRP stationary over the stance foot during the single support phase, and using a linear interpolation for the transition from one foot to the other during the double support phase. As a terminal constraint, the final DCM position $\boldsymbol{\xi}_{f}^{d}$ is chosen to coincide with the final VRP waypoint, i.e. the DCM is in equilibrium at the end of the trajectory $\left(\dot{\boldsymbol{\xi}}_{f}^{d}=0\right.$, see (3)). To ensure the continuity of the trajectories during the transition from standing to walking, an additional VRP waypoint is computed and inserted into the sequence after the first waypoint (more details are given in [10] and [11]).

\section{B. Continuous foot reference trajectories}

The swing foot trajectories are generated using piecewise fifth-order polynomials for each Cartesian coordinate $(x, y$, $z$, roll, pitch, yaw). The vertical motion is implemented using two fifth-order polynomials, one for the upward, the other for the downward movement.

For walking with edge contacts, the reference foot trajectories are generated as follows: First, while the foot is in contact with the ground, the center of pressure (CoP) is gradually shifted towards the front of the foot by suitably changing the contact constraints for the wrench distribution solver. Then, when the CoP reaches the edge of the foot, the foot pivots on its front edge while maintaining an edge contact with the ground. The foot pitch trajectory is also implemented as a fifth-order polynomial. The foot is lifted from the ground at the moment of maximum angular velocity, which corresponds to the maximum translational velocity of the foot center, leading to a fluent foot motion. Currently, our edge contact trajectory does not use a heel contact, as we found that the kinematic benefit does not compensate for the reduction in the contact wrench during contact acquisition, and the overall slower motion of the foot due to the additional phases (pivoting, CoP transition).

\section{WHOLE-BODY TORQUE CONTROLLER}

In this work, following the suggestion in [23], we describe the dynamics of a humanoid robot as a floating base model using the CoM position ${ }^{1} \boldsymbol{x}_{c} \in \mathbb{R}^{3}$ and the waist orientation $\boldsymbol{R}_{c} \in \mathcal{S O}(3)$. The corresponding linear and angular velocities, $\dot{\boldsymbol{x}}_{c}$ and $\boldsymbol{\omega}_{c} \in \mathbb{R}^{3}$, are stacked into a velocity vector $\boldsymbol{\nu}_{c}=\left(\dot{\boldsymbol{x}}_{c}^{T} \boldsymbol{\omega}_{c}^{T}\right)^{T}$. Based on the joint angles $\boldsymbol{q} \in \mathbb{R}^{n}$ for the $n$ actuated joints, the equations of motion can be written as

$$
\boldsymbol{M}\left(\begin{array}{c}
\dot{\boldsymbol{\nu}}_{c} \\
\ddot{\boldsymbol{q}}
\end{array}\right)+\boldsymbol{C}\left(\begin{array}{c}
\boldsymbol{\nu}_{c} \\
\dot{\boldsymbol{q}}
\end{array}\right)+\left(\begin{array}{c}
-\boldsymbol{w}_{g} \\
\mathbf{0}
\end{array}\right)=\left(\begin{array}{c}
\mathbf{0} \\
\boldsymbol{\tau}
\end{array}\right)+\overline{\boldsymbol{\tau}}_{\text {ext }},
$$

\footnotetext{
${ }^{1}$ Unless mentioned otherwise, all position vectors and orientations are given with respect to the world frame.
} 


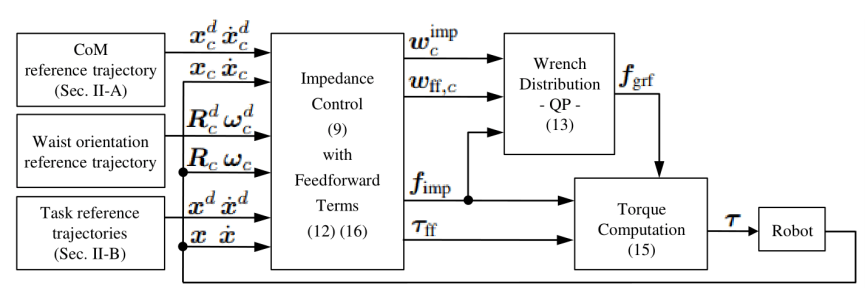

Fig. 2: Overview of the whole-body torque controller

where $M$ and $C$ represent the inertia and Coriolis matrices, respectively, and $\boldsymbol{w}_{g}=\left(m \boldsymbol{g}^{T} \mathbf{0}^{T}\right)^{T}$ denotes the gravitational wrench, with $m$ being the total mass of the robot, and $\boldsymbol{g} \in$ $\mathbb{R}^{3}$ the gravitational acceleration vector. Here, $\tau \in \mathbb{R}^{n}$ is the vector of actuator torques, while $\overline{\boldsymbol{\tau}}_{\text {ext }} \in \mathbb{R}^{\bar{n}}$ denotes the generalized external forces, with $\bar{n}=n+6$ being the total number of degrees of freedom (DoF) for the humanoid robot.

In [21], the model is transformed into Cartesian task space by replacing the joint coordinates with the Cartesian coordinates of the end-effectors, while in [24], generalized tasks are combined into a task hierarchy using null space projections.

\section{A. Generalized controller formulation}

Let $\boldsymbol{x}_{i} \in \mathbb{R}^{m_{i}}$ be the coordinates of $k$ operational space tasks, with $m_{i}$ denoting the dimension of the $i$-th task. The corresponding task velocities are described by the Jacobian matrices $\boldsymbol{J}_{i} \in \mathbb{R}^{m_{i} \times \bar{n}}$. In this work, we consider that all DoF are covered by the task definitions, such that $\sum_{i=1}^{k} m_{i}=\bar{n}$. Stacking all generalized task velocities, we obtain

$$
\underbrace{\left(\begin{array}{c}
\dot{\boldsymbol{x}}_{1} \\
\vdots \\
\dot{\boldsymbol{x}}_{k}
\end{array}\right)}_{\dot{\boldsymbol{x}} \in \mathbb{R}^{\bar{n}}}=\underbrace{\left[\begin{array}{c}
\boldsymbol{J}_{1} \\
\vdots \\
\boldsymbol{J}_{k}
\end{array}\right]}_{\boldsymbol{J} \in \mathbb{R}^{\bar{n} \times \bar{n}}}\left(\begin{array}{c}
\boldsymbol{\nu}_{c} \\
\dot{\boldsymbol{q}}
\end{array}\right) \text {. }
$$

As we are working in the context of legged robots, we consider the following tasks to be predefined: a 6 -dimensional tracking task for the robot base (the CoM location and the waist orientation), and a task of size $m_{\text {grf }}$ for generating contact forces subject to the contact constraints. ${ }^{2}$ Note that $m_{\text {grf }}$ is not a constant value, but depends on the current contact configuration. ${ }^{3}$ We combine the remaining tasks into a generalized task of size $m_{\text {imp }}=n-m_{\text {grf }} .4$ This can contain Cartesian tracking tasks for the free end-effectors, joint space subtasks, visual servoing tasks, etc. In light of these considerations, we can rewrite (5) as

$$
\underbrace{\left(\begin{array}{c}
\boldsymbol{\nu}_{c} \\
\dot{\boldsymbol{x}}_{\mathrm{grf}} \\
\dot{\boldsymbol{x}}_{\mathrm{imp}}
\end{array}\right)}_{\dot{\boldsymbol{x}}}=\underbrace{\left[\begin{array}{cc}
\boldsymbol{I}_{6 \times 6} & \mathbf{0}_{6 \times n} \\
\boldsymbol{J}_{\mathrm{grf}, c} & \boldsymbol{J}_{\mathrm{grf}, q} \\
\boldsymbol{J}_{\mathrm{imp}, c} & \boldsymbol{J}_{\mathrm{imp}, q}
\end{array}\right]}_{\boldsymbol{J}}\left(\begin{array}{c}
\boldsymbol{\nu}_{c} \\
\dot{\boldsymbol{q}}
\end{array}\right),
$$

\footnotetext{
2"grf" stands for ground reaction force. In this paper, we use the term in a more general sense to include forces created by the hands or elbows in contact with the environment.

${ }^{3}$ For example, during walking, in the single support phase $m_{\text {grf }}=6$, while in double support $m_{\text {grf }}=12$, assuming full contacts with the ground. In case of walking with edge contacts, $m_{\text {grf }}$ can take any value in the interval $[6,12]$.

${ }^{4}$ here, "imp" stands for impedance.
}

where $\boldsymbol{J}_{\mathrm{grf}}=\left[\boldsymbol{J}_{\mathrm{grf}, c} \boldsymbol{J}_{\mathrm{grf}, q}\right] \in \mathbb{R}^{m_{\mathrm{grr}} \times \bar{n}}$ is the Jacobian matrix for the contact task, while $\boldsymbol{J}_{\text {imp }}=\left[\boldsymbol{J}_{\text {imp }, c} \boldsymbol{J}_{\text {imp }, q}\right] \in$ $\mathbb{R}^{m_{\text {imp }} \times \bar{n}}$ is the Jacobian matrix for the remaining generalized tasks. We assume that the matrix $\boldsymbol{J}$ is invertible, leaving redundancy and singularity considerations for future work. Then, given a reference task velocity $\dot{\boldsymbol{x}}^{d}$, the corresponding references for the CoM and joint velocities can be computed via inverse kinematics:

$$
\left(\begin{array}{c}
\boldsymbol{\nu}_{c}^{d} \\
\dot{\boldsymbol{q}}^{d}
\end{array}\right)=\boldsymbol{J}^{-1} \dot{\boldsymbol{x}}^{d}
$$

Note that in (7), the Jacobian matrix $\boldsymbol{J}$ is the same as in (6), i.e. the Jacobian is computed at each time instant from actual values, and not from references.

Similar to the approach taken in [21], we choose a closedloop system behavior inspired by the PD+ control for a robotic manipulator [25]. Using the floating base model (4) and the generalized tasks described in (6), we write the desired closed-loop dynamics as

$$
\boldsymbol{M}\left(\begin{array}{c}
\Delta \dot{\boldsymbol{\nu}}_{c} \\
\Delta \ddot{\boldsymbol{q}}
\end{array}\right)+\boldsymbol{C}\left(\begin{array}{c}
\Delta \boldsymbol{\nu}_{c} \\
\Delta \dot{\boldsymbol{q}}
\end{array}\right)=\overline{\boldsymbol{\tau}}_{e x t}-\boldsymbol{J}^{T}\left(\begin{array}{c}
\boldsymbol{w}_{c}^{\mathrm{imp}} \\
\boldsymbol{f}_{\mathrm{grf}} \\
\boldsymbol{f}_{\mathrm{imp}}
\end{array}\right),
$$

where $\Delta \boldsymbol{\nu}_{c}=\boldsymbol{\nu}_{c}-\boldsymbol{\nu}_{c}^{d}$ and $\Delta \dot{\boldsymbol{q}}=\dot{\boldsymbol{q}}-\dot{\boldsymbol{q}}^{d}$ represent the deviations from the reference trajectories. The contact forces $f_{\text {grf }} \in \mathbb{R}^{m_{\text {grf }}}$ are subject to contact constraints and will be computed in a subsequent step using a quadratic programming $(\mathrm{QP})$ approach, while the impedance forces $f_{\text {imp }} \in \mathbb{R}^{m_{\text {imp }}}$ are given by the corresponding tasks. In (8), $\boldsymbol{w}_{c}^{\text {imp }}$ denotes the CoM impedance wrench, which is computed as

$$
\boldsymbol{w}_{c}^{\mathrm{imp}}=\left(\begin{array}{c}
\boldsymbol{K}_{c}\left(\boldsymbol{x}_{c}-\boldsymbol{x}_{c}^{d}\right)+\boldsymbol{D}_{c}\left(\dot{\boldsymbol{x}}_{c}-\dot{\boldsymbol{x}}_{c}^{d}\right) \\
\boldsymbol{\tau}_{r}\left(\boldsymbol{\Sigma}_{c}, \boldsymbol{R}_{c}^{T} \boldsymbol{R}_{c}^{d}\right)+\boldsymbol{B}_{c}\left(\boldsymbol{\omega}_{c}-\boldsymbol{\omega}_{c}^{d}\right)
\end{array}\right) .
$$

It consists of a linear spring-damper system for controlling the CoM location, with positive diagonal matrices $\boldsymbol{K}_{c}>0$ and $\boldsymbol{D}_{c}>0$, and a rotational spring $\boldsymbol{\tau}_{r}$ [26] with stiffness $\boldsymbol{\Sigma}_{c}>0$ combined with a rotational damper $\boldsymbol{B}_{c}>0$ for controlling the waist orientation. The impedance forces $f_{\text {imp }}$ are computed similarly for Cartesian tasks or via a simple joint impedance law.

Combining (4) with (8) and rearranging the terms, we obtain an equation with two unknowns, the contact forces $f_{\text {grf }}$ and the joint torques $\tau$ :

$$
\underbrace{\left[\begin{array}{c}
\boldsymbol{M}_{1} \\
\boldsymbol{M}_{2}
\end{array}\right]}_{\boldsymbol{M}}\left(\begin{array}{c}
\dot{\boldsymbol{\nu}}_{c}^{d} \\
\ddot{\boldsymbol{q}}^{d}
\end{array}\right)+\underbrace{\left[\begin{array}{c}
\boldsymbol{C}_{1} \\
\boldsymbol{C}_{2}
\end{array}\right]}_{\boldsymbol{C}}\left(\begin{array}{c}
\boldsymbol{\nu}_{c}^{d} \\
\dot{\boldsymbol{q}}^{d}
\end{array}\right)=\left(\begin{array}{c}
\boldsymbol{w}_{g} \\
\boldsymbol{\tau}
\end{array}\right)+\boldsymbol{J}^{T}\left(\begin{array}{c}
\boldsymbol{w}_{c}^{\text {imp }} \\
\boldsymbol{f}_{\mathrm{grf}} \\
\boldsymbol{f}_{\text {imp }}
\end{array}\right) .
$$

It is straightforward to observe that the upper 6 rows of (10) are independent of $\tau$, which allows us to write the following equality for the contact forces $f_{\mathrm{grf}}$ :

$$
\boldsymbol{J}_{\mathrm{grf}, c}^{T} \boldsymbol{f}_{\mathrm{grf}}=\boldsymbol{w}_{\mathrm{ff}, c}-\boldsymbol{w}_{g}-\boldsymbol{w}_{c}^{\mathrm{imp}}-\boldsymbol{J}_{\mathrm{imp}, c}^{T} \boldsymbol{f}_{\mathrm{imp}},
$$

where

$$
\boldsymbol{w}_{\mathrm{ff}, c}=\boldsymbol{M}_{1}\left(\begin{array}{c}
\dot{\boldsymbol{\nu}}_{c}^{d} \\
\ddot{\boldsymbol{q}}^{d}
\end{array}\right)+\boldsymbol{C}_{1}\left(\begin{array}{c}
\boldsymbol{\nu}_{c}^{d} \\
\dot{\boldsymbol{q}}^{d}
\end{array}\right)
$$


and we used the structure of $\boldsymbol{J}$ from (6). We can now obtain $f_{\text {grf }}$ as a solution of the constrained QP problem

$$
\min _{\boldsymbol{f}_{\mathrm{grf}}}\left(\frac{1}{2} \boldsymbol{\delta}_{c}^{T} \boldsymbol{Q}_{c} \boldsymbol{\delta}_{c}+\frac{1}{2} \boldsymbol{\delta}_{f}^{T} \boldsymbol{Q}_{f} \boldsymbol{\delta}_{f}\right)
$$

with the residuals

$$
\begin{aligned}
& \boldsymbol{\delta}_{c}=\boldsymbol{J}_{\mathrm{grf}, c}^{T} \boldsymbol{f}_{\mathrm{grf}}-\boldsymbol{w}_{\mathrm{ff}, c}+\boldsymbol{w}_{g}+\boldsymbol{w}_{c}^{\mathrm{imp}}+\boldsymbol{J}_{\mathrm{imp}, c}^{T} \boldsymbol{f}_{\mathrm{imp}} \\
& \boldsymbol{\delta}_{f}=\boldsymbol{f}_{\mathrm{grf}}-\boldsymbol{f}_{\mathrm{grf}}^{d}
\end{aligned}
$$

and subject to contact constraints (contact unilaterality, friction cone constraints, center of pressure constraints, bounded normal force, and bounded torque on the $z$-axis). In (14), $\boldsymbol{f}_{\mathrm{grf}}^{d}$ represents an optional precomputed solution for the contact forces, which could be provided by a motion planner. If no such solution is available, we choose by default $\boldsymbol{f}_{\text {grf }}^{d}=\mathbf{0}$, leading to $\boldsymbol{\delta}_{f}$ acting as a $L_{2}$ regularization (i.e. minimizing the Euclidean norm of $\boldsymbol{f}_{\text {grf }}$ weighted by $\boldsymbol{Q}_{f}$ ). Here, $\boldsymbol{Q}_{c}$ and $\boldsymbol{Q}_{f}$ are positive diagonal matrices, chosen such that $\boldsymbol{Q}_{c} \gg \boldsymbol{Q}_{f}$, thereby giving a higher priority to the CoM task compared to the contact force minimization task.

We choose to implement (11) using a soft constraint in (13) instead of a hard constraint. Using a hard constraint would be equivalent to solving (11) with a weighted pseudoinverse of $\boldsymbol{J}_{\mathrm{grf}, c}^{T}$, similar to the approach taken in [27]. During our walking experiments over uneven terrain, we found that, depending on the magnitude of the perturbation, the contact constraints and the CoM task cannot be fulfilled simultaneously throughout the motion. In this case, it is preferable to have a partial solution for the CoM task instead of the QP solver returning no result, especially since these situations are mostly transient, and the controller can recover the accumulated drift from the CoM reference trajectory.

Comparing (13) to the QP problem definition in [21], note that in this work only the contact forces $f_{\text {grf }}$ are selected as optimization variables, not the complete vector of forces $\left(\boldsymbol{f}_{\mathrm{grf}}^{T} \boldsymbol{f}_{\mathrm{imp}}^{T}\right)^{T}$ as in [21]. When reaching the boundaries of the contact constraints, the QP solver in [21] tries to generate additional wrenches in the free end-effectors, overriding the impedance tasks, in order to solve the CoM task. However, as these end-effectors are not in contact with the environment, the additional commanded end-effector wrenches lead to jerky motions and reduced robustness of the overall locomotion task. An additional advantage of our method is that it reduces the number of $\mathrm{QP}$ weights, and therefore, the parameter tuning effort.

Finally, having obtained the contact forces $f_{\text {grf }}$ from the optimization solver, we use the bottom rows of (10) to compute the commanded joint torques:

$$
\boldsymbol{\tau}=\boldsymbol{\tau}_{\mathrm{ff}}-\left[\begin{array}{ll}
\boldsymbol{J}_{\mathrm{grf}, q}^{T} & \boldsymbol{J}_{\mathrm{imp}, q}^{T}
\end{array}\right]\left(\begin{array}{c}
\boldsymbol{f}_{\mathrm{grf}} \\
\boldsymbol{f}_{\mathrm{imp}}
\end{array}\right),
$$

where

$$
\boldsymbol{\tau}_{\mathrm{ff}}=\boldsymbol{M}_{2}\left(\begin{array}{c}
\dot{\boldsymbol{\nu}}_{c}^{d} \\
\ddot{\boldsymbol{q}}^{d}
\end{array}\right)+\boldsymbol{C}_{2}\left(\begin{array}{c}
\boldsymbol{\nu}_{c}^{d} \\
\dot{\boldsymbol{q}}^{d}
\end{array}\right)
$$

\section{B. DCM tracking control}

In this section, we show that a pure DCM tracking controller can be implemented within the generalized controller formulation. Let $\boldsymbol{w}_{c}$ denote the total wrench acting on the CoM, as computed by the controller in (11). It considers the effects of the commanded ground reaction and impedance forces, and includes the gravity wrench:

$$
\boldsymbol{w}_{c}=\boldsymbol{J}_{\mathrm{grf}, c}^{T} \boldsymbol{f}_{\mathrm{grf}}+\boldsymbol{J}_{\mathrm{imp}, c}^{T} \boldsymbol{f}_{\mathrm{imp}}+\boldsymbol{w}_{g} .
$$

Using (17) and the known structure of the inertia and Coriolis matrices [28], we can rewrite (11) as

$$
\begin{aligned}
\underbrace{\left(\begin{array}{c}
\boldsymbol{f}_{c} \\
\boldsymbol{\tau}_{c}
\end{array}\right)}_{\boldsymbol{w}_{c}}= & \underbrace{\left[\begin{array}{ccc}
m \boldsymbol{I} & \mathbf{0} & \mathbf{0} \\
\mathbf{0} & \boldsymbol{M}_{1 \omega} & \boldsymbol{M}_{1 q}
\end{array}\right]}_{\boldsymbol{M}_{1}}\left(\begin{array}{c}
\ddot{\boldsymbol{x}}_{c}^{d} \\
\dot{\boldsymbol{\omega}}_{c}^{d} \\
\ddot{\boldsymbol{q}}^{d}
\end{array}\right) \\
& +\underbrace{\left[\begin{array}{ccc}
\mathbf{0} & \mathbf{0} & \mathbf{0} \\
\mathbf{0} & \boldsymbol{C}_{1 \omega} & \boldsymbol{C}_{1 q}
\end{array}\right]}_{\boldsymbol{C}_{1}}\left(\begin{array}{c}
\dot{\boldsymbol{x}}_{c}^{d} \\
\boldsymbol{\omega}_{c}^{d} \\
\dot{\boldsymbol{q}}^{d}
\end{array}\right)-\underbrace{\left(\begin{array}{c}
\boldsymbol{f}_{c}^{\text {imp }} \\
\boldsymbol{\tau}_{c}^{\text {imp }}
\end{array}\right)}_{\boldsymbol{w}_{c}^{\text {imp }}} .
\end{aligned}
$$

Note that, similarly to [28], it is always possible to choose a Coriolis matrix factorization for which the first three rows and columns of $\boldsymbol{C}$ are identically zero [29]. The top 3 lines of (18) represent the closed-loop CoM dynamics

$$
m \ddot{\boldsymbol{x}}_{c}=m \ddot{\boldsymbol{x}}_{c}^{d}-\boldsymbol{K}_{c}\left(\boldsymbol{x}_{c}-\boldsymbol{x}_{c}^{d}\right)-\boldsymbol{D}_{c}\left(\dot{\boldsymbol{x}}_{c}-\dot{\boldsymbol{x}}_{c}^{d}\right) .
$$

Introducing a coupling between the gain matrices $\boldsymbol{K}_{c}$ and $\boldsymbol{D}_{c}$ of the form

$$
\boldsymbol{D}_{c}=\frac{m}{b} \boldsymbol{I}+b \boldsymbol{K}_{c}
$$

and rewriting (19) using the DCM definition (1) (also applied to the reference quantities, i.e. $\boldsymbol{\xi}^{d}:=\boldsymbol{x}_{c}^{d}+b \dot{\boldsymbol{x}}_{c}^{d}$ ), we obtain the stable closed-loop DCM error dynamics

$$
\dot{\boldsymbol{\xi}}-\dot{\boldsymbol{\xi}}^{d}=-\frac{b}{m} \boldsymbol{K}_{c}\left(\boldsymbol{\xi}-\boldsymbol{\xi}^{d}\right) .
$$

Therefore, we can conclude that, given the coupling of the gain matrices $\boldsymbol{K}_{c}$ and $\boldsymbol{D}_{c}$, a pure DCM controller [7] with the DCM error gain $\boldsymbol{K}_{\xi}=\frac{b}{m} \boldsymbol{K}_{c}$ can be implemented within the generalized whole-body controller.

\section{DYNAMIC WALKING IMPLEMENTATION}

The framework presented in the previous section can be directly applied to implement a dynamic walking controller. In this section, we present the controller implementation details, discussing an additional source of torque discontinuities caused by contact transitions and the approach taken to minimize them. Additionally, we introduce a stance foot stabilization method, which is particularly relevant when walking over compliant or rough terrain.

\section{A. Task formulation for dynamic walking}

In this work, for dynamic walking, we focus on the CoM trajectory and the Cartesian foot trajectories. Let $\boldsymbol{\nu}_{r}$ and $\boldsymbol{\nu}_{l}$ denote the right and left foot Cartesian velocity vectors, respectively, which are defined in an analogous manner to the CoM velocity vector $\nu_{c}$. The CoM and foot tasks account 
for 18 assigned DoF that are formulated as subtasks in the whole-body task formulation. For the remaining $\bar{n}-18 \mathrm{DoF}$, corresponding to the upper body of the humanoid robot, we add a joint space task for maintaining a given body posture. We can now rewrite (5) in a particular form that maps the generalized velocities to walking-related task velocities:

$$
\underbrace{\left(\begin{array}{c}
\boldsymbol{\nu}_{c} \\
\boldsymbol{\nu}_{r} \\
\boldsymbol{\nu}_{l} \\
\dot{\boldsymbol{q}}_{\text {pose }}
\end{array}\right)}_{\dot{\boldsymbol{x}}}=\underbrace{\left[\begin{array}{cc}
\boldsymbol{I} & \mathbf{0} \\
\boldsymbol{J}_{r, c} & \boldsymbol{J}_{r, q} \\
\boldsymbol{J}_{l, c} & \boldsymbol{J}_{l, q} \\
\mathbf{0} & \boldsymbol{S}_{\text {pose }}
\end{array}\right]}_{\boldsymbol{J}}\left(\begin{array}{c}
\boldsymbol{\nu}_{c} \\
\dot{\boldsymbol{q}}
\end{array}\right),
$$

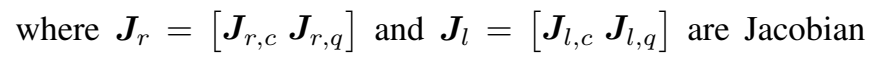
matrices for the right and left foot, respectively, while $S_{\text {pose }} \in \mathbb{R}^{(n-12) \times n}$ is a selection matrix for the joint space posture task. Depending on the contact configuration, $\dot{\boldsymbol{x}}_{\mathrm{grf}}$ and $\dot{\boldsymbol{x}}_{\text {imp }}$ from (6) can be assigned to the corresponding elements of the walking task velocities $\nu_{r}, \nu_{l}$, and $\dot{\boldsymbol{q}}_{\text {pose }}$ : during the double support phase, $\dot{\boldsymbol{x}}_{\text {grf }}=\left(\begin{array}{ll}\boldsymbol{\nu}_{r}^{T} & \boldsymbol{\nu}_{l}^{T}\end{array}\right)^{T}$ and $\dot{\boldsymbol{x}}_{\mathrm{imp}}=\dot{\boldsymbol{q}}_{\text {pose }}$; during the single support phase, either $\dot{\boldsymbol{x}}_{\mathrm{grf}}=\boldsymbol{\nu}_{r}$ and $\dot{\boldsymbol{x}}_{\mathrm{imp}}=\left(\begin{array}{ll}\boldsymbol{\nu}_{l}^{T} & \dot{\boldsymbol{q}}_{\text {pose }}^{T}\end{array}\right)^{T}$, or $\dot{\boldsymbol{x}}_{\mathrm{grf}}=\boldsymbol{\nu}_{l}$ and $\dot{\boldsymbol{x}}_{\text {imp }}=\left(\boldsymbol{\nu}_{r}^{T} \dot{\boldsymbol{q}}_{\text {pose }}^{T}\right)^{T}$, depending on the contact foot. In all cases, however, we can rewrite (15) for dynamic walking as

$$
\boldsymbol{\tau}=\boldsymbol{\tau}_{\mathrm{ff}}-\boldsymbol{S}_{\text {pose }}^{T} \boldsymbol{\tau}_{\text {pose }}-\left[\begin{array}{ll}
\boldsymbol{J}_{r, q}^{T} & \boldsymbol{J}_{l, q}^{T}
\end{array}\right]\left(\begin{array}{c}
\boldsymbol{w}_{r} \\
\boldsymbol{w}_{l}
\end{array}\right),
$$

where $\boldsymbol{\tau}_{\text {pose }}$ denotes the posture impedance torque, while $\boldsymbol{w}_{r}$ and $\boldsymbol{w}_{l}$ are the right and left foot wrenches, respectively.

\section{B. Continuous foot wrench transitions}

An additional source of torque discontinuities is given by the discrete contact transitions. Consider the right foot wrench $\boldsymbol{w}_{r}$ (the left foot wrench $\boldsymbol{w}_{l}$ is treated similarly): during the right stance phase it is computed by the QP solver according to the CoM task and the contact constraints, while during the corresponding swing phase it is computed as an impedance wrench by the foot tracking task. A discontinuous switch from one control scheme to the other may change the foot wrench drastically, leading to jumps in the commanded torques (see (23)). To prevent foot wrench discontinuities, we introduce one transition phase at the beginning and another one at the end of the stance phase, each with a fixed duration $T_{a}$. During the transition phases, the foot wrench is computed by the QP solver, but the contact constraints are continuously adjusted to ensure wrench continuity.

In the following, we use the contact normal force $f_{z}$ as a clarifying example. The corresponding contact constraints are: maximum normal force $\left(f_{z} \leqslant \bar{f}_{z}\right)$, and minimum normal force $\left(f_{z} \geqslant \underline{f}_{z} \geqslant 0\right.$, designed to ensure contact stability and guarantee contact unilaterality). Let $z$ and $\dot{z}$ be the vertical position and velocity of the foot, respectively, expressed in the contact frame, with $z^{d}$ and $\dot{z}^{d}$ the corresponding reference values. Then, we can write the impedance force for the $z$-axis as

$$
f_{z}^{\mathrm{imp}}=k_{z}\left(z-z^{d}\right)+d_{z}\left(\dot{z}-\dot{z}^{d}\right),
$$

where $k_{z}>0$ and $d_{z}>0$ denote the stiffness and damping gains, respectively. Note that, in general, at the end of the swing phase $f_{z}^{\text {imp }} \neq 0$ due to tracking errors. When walking on compliant or uneven terrain, this is often caused by the terrain irregularities.

Finally, during the transition from the swing to the stance phase $^{5}$, we use the following contact lower and upper bounds

$$
\begin{aligned}
& \underline{f}_{z}^{a}=\frac{t}{T_{a}} \underline{f}_{z}+\left(1-\frac{t}{T_{a}}\right) f_{z}^{\mathrm{imp}} \\
& \bar{f}_{z}^{a}=\frac{t}{T_{a}} \bar{f}_{z}+\left(1-\frac{t}{T_{a}}\right) f_{z}^{\mathrm{imp}},
\end{aligned}
$$

where $t \in\left[0, T_{a}\right]$ is the local time of the transition phase. Since the QP solver is constrained to finding a solution for $f_{z}$ within the interval $\left[f_{z}^{a}, \bar{f}_{z}^{a}\right]$, our approach guarantees that at the beginning of the transition phase $f_{z}=f_{z}^{\mathrm{imp}}$, and during the transition phase the constraint interval is gradually widened to reach the default interval $\left[\underline{f}_{z}, \bar{f}_{z}\right]$ at the end of the transition phase. Similar constraints are applied on the remaining elements of the foot wrench $\boldsymbol{w}_{r}$. Figure 3 shows the effect of the presented approach while walking over compliant terrain (the transition phase duration $T_{a}$ is $0.1 \mathrm{~s}, \underline{f}_{z}=50 \mathrm{~N}$, and $\left.\bar{f}_{z}=900 \mathrm{~N}\right)$.

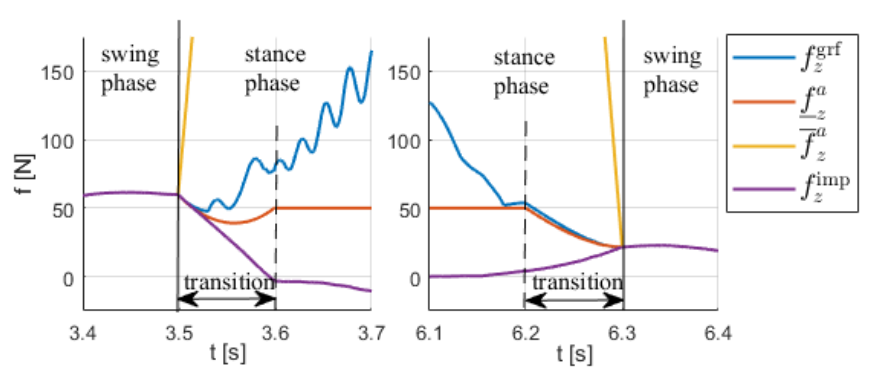

Fig. 3: Force continuity while walking over compliant terrain. Left: transition from swing to stance phase starts at $3.5 \mathrm{~s}$. Right: transition from stance to swing phase starts at $6.2 \mathrm{~s}$.

\section{Stance foot stabilization}

One of the challenges of walking on compliant or uneven terrain is that a rigid contact with the ground surface can no longer be guaranteed. This means that the contact foot can have non-zero linear velocity (e.g. sink into the mattress) or can rotate, leading to foot oscillations.

In our passivity-based controller described above, the contact foot is controlled solely through the contact wrench computed by the QP solver, with no additional task for maintaining a certain foot position or orientation. For example, when the right foot is in contact (the left foot is treated analogously), the corresponding wrench $\boldsymbol{w}_{r}$ is computed as part of the vector $f_{\text {grf }}$. To stabilize the foot, we add a damping wrench $\boldsymbol{w}_{r}^{\text {stabil }}=-\boldsymbol{D} \boldsymbol{\nu}_{r}$ to $\boldsymbol{w}_{r}$ in (23), with $\boldsymbol{D}$ as a positive diagonal damping matrix. During the transition phases introduced in the previous section, the damping gain $\boldsymbol{D}$ is adjusted in order to prevent torque discontinuities.

\footnotetext{
${ }^{5}$ The transition from stance to swing phase is handled analogously.
} 
The stabilization wrench acts only if the stance foot moves, i.e. if the rigid contact assumption is violated. Otherwise, the stabilization wrench is zero. If the stance foot moves, the damping wrenches $\boldsymbol{w}_{r}^{\text {stabil }}$ and $\boldsymbol{w}_{l}^{\text {stabil }}$ act as disturbances to the CoM task. However, in this case, the CoM task cannot be fulfilled anyway, as it relies on the assumption of a rigid contact to produce the required forces. Therefore, the temporary additional disturbance to the CoM caused by the damping wrenches leading to the subsequent stabilization of the stance foot has a reduced net effect on the overall tracking performance. While walking on a compliant surface (gym mattress), we found that the proposed stabilization method significantly reduces the contact foot oscillations without notably compromising the CoM tracking task (see Fig. 4).
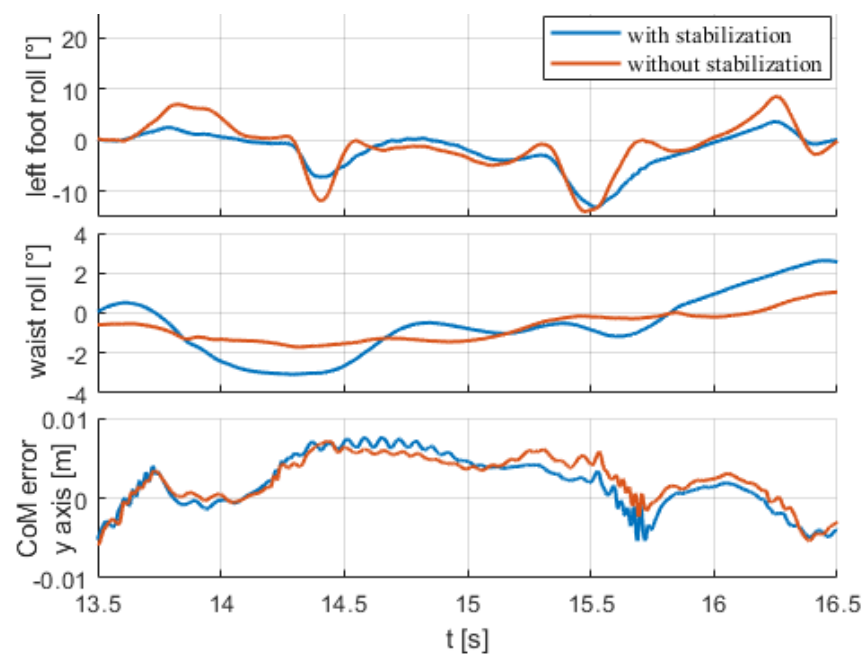

Fig. 4: Left foot stabilization during the stance phase.

\section{EXPERIMENTAL EVALUATION}

We performed extensive experiments with the torquecontrolled humanoid robot TORO [15] walking on various terrains (flat terrain, grass, gravel, gym mattress). Videos of the experiments can be found in the multimedia attachment.

\section{A. Robotic system}

TORO is a $1.74 \mathrm{~m}$ tall humanoid robot, weighing $76.4 \mathrm{~kg}$ [15]. It has 25 torque-controllable joints (six in each limb, and one for vertical torso rotation), 2 position-controlled joints in the neck, and is equipped with multiple sensors: position and torque sensors in each joint, an inertial measurement unit (IMU) in the torso, force-torque sensors (FTS) at the feet, and stereo and depth perception cameras in the head. Our controller implementation uses only the joint position sensors and the IMU for state estimation [21], the FTS data is not used in the control loop.

The controller and the trajectory generator are implemented in Matlab/Simulink, using Simulink Coder for generating real-time code, and qpOASES [30] for solving the constrained QP problem (13). The whole-body controller is executed at a rate of $1 \mathrm{kHz},{ }^{6}$ while the low-level joint torque

\footnotetext{
${ }^{6}$ the total execution time including the computation of the dynamics matrices $\boldsymbol{M}$ and $\boldsymbol{C}$ is $700 \mu$ s.
}

controller is executed at a rate of $3 \mathrm{kHz}$ [31].

The walking timing parameters (single and double support times) were chosen for each experiment by taking into account the stepping parameters (step length and height) and the walking surface. Initially, the CoM damping gain $\boldsymbol{D}_{c}$ was chosen according to (20), however, the first experiments showed that friction within the robotic system requires a reduction of this gain. For the presented experiments, the damping gain was reduced by a factor of 2 .

\section{B. Experiments}

The reference and actual trajectories on the $x$ and $y$ axes for the feet, the DCM, and the CoM, are shown in Figure 5, for three different experiments.

The first set of experiments we performed with the presented trajectory generation and whole-body controller was dynamic walking on flat terrain, where we iteratively reduced the step time (i.e. increased the step frequency) and increased the step length. The maximum step frequency we achieve with $15 \mathrm{~cm}$ steps is 1 step per second (single/double support time: $0.7 / 0.3 \mathrm{~s}$ ), corresponding to a walking speed of 0.15 $\mathrm{m} / \mathrm{s}$. Using edge contacts, we are able to increase the step length significantly, and achieve a speed of $0.37 \mathrm{~m} / \mathrm{s}$ while walking with $55 \mathrm{~cm}$ steps and $1.5 \mathrm{~s}$ step time (single/double support time: $1.1 / 0.4 \mathrm{~s}$ ), see Figure 5a. Note the foot tracking performance of the controller: for a stride length of 110 $\mathrm{cm}$ and a swing phase duration of 1.1 seconds, the foot placement error at touchdown is less that $3 \mathrm{~cm}$, while the maximum error during the motion is less that $6 \mathrm{~cm}$.

The second set of experiments evaluates the performance of our approach in tracking the CoM trajectory while walking over uneven terrain (grass). For this, the robot was taken outdoors and commanded to walk over a grassy field; throughout the experiment, the robot has no knowledge of the terrain and assumes a level ground. Figure 5b shows the CoM tracking performance while walking with $15 \mathrm{~cm}$ steps and $1.2 \mathrm{~s}$ step time (single/double support time: $0.9 / 0.3 \mathrm{~s}$ ): the mean tracking error of the DCM is $13 \mathrm{~mm}$ on the $x$-axis and 7 $\mathrm{mm}$ on the $y$-axis (the mean tracking error of the CoM is $13 \mathrm{~mm}$ on the $x$-axis and $4 \mathrm{~mm}$ on the $y$-axis). With our presented approach, TORO is also capable of walking on grass with edge contacts, taking $25 \mathrm{~cm}$ steps with $1.7 \mathrm{~s}$ step time (single/double support time: $1.2 / 0.5 \mathrm{~s}$ ).

The third set of experiments tests the robustness of our approach against a soft, compliant ground surface. For this purpose, a gym mattress of size $1 \mathrm{~m} \times 2 \mathrm{~m} \times 8 \mathrm{~cm}$ made of a compound foam with a density of $20 \mathrm{~kg} / \mathrm{m}^{3}$ was placed on the ground in front of the robot. This represents, to the best of our knowledge, the first report of a humanoid robot walking over a soft gym mattress. As the robot has no knowledge of the ground, to avoid stumbling when stepping onto the mattress and prevent dragging the feet while walking, the step height is set to $15 \mathrm{~cm}$ (from the default value of $5 \mathrm{~cm}$ ). We performed the first trial with stance foot stabilization: the robot is commanded to walk forward with $10 \mathrm{~cm}$ steps and $2 \mathrm{~s}$ step time (single/double support time: $1.2 / 0.8 \mathrm{~s}$ ). Figure $5 \mathrm{c}$ shows the last 18 seconds of the experiment. Note the 

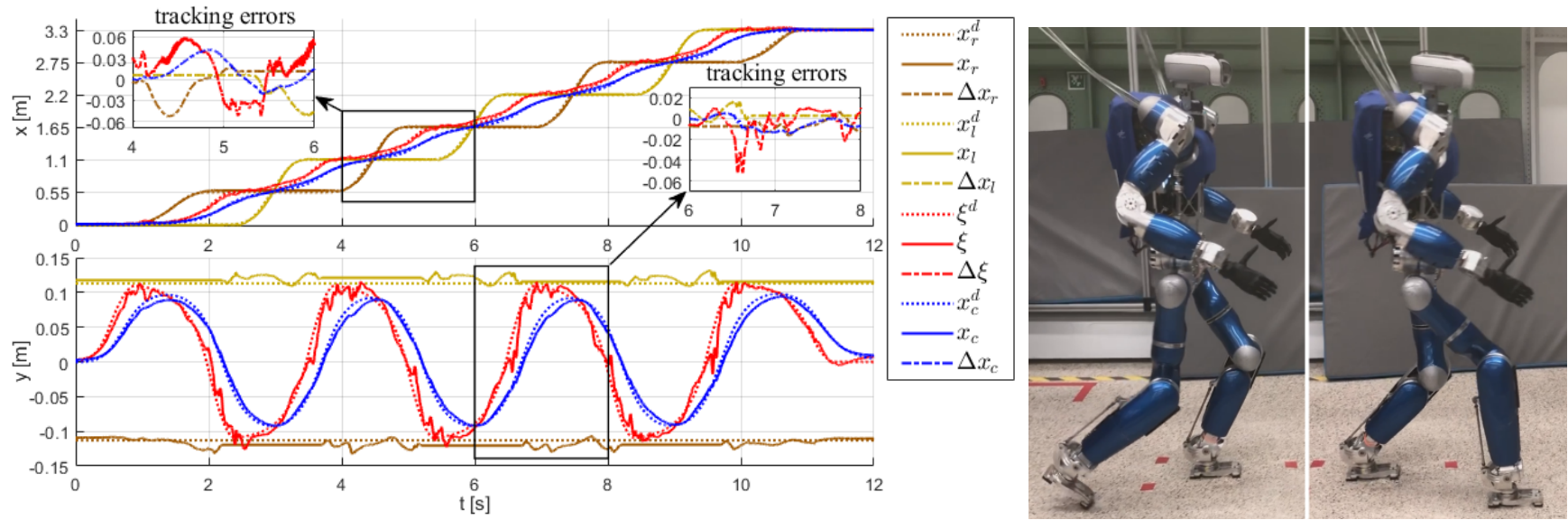

(a) Walking on flat terrain with edge contacts (step length: $55 \mathrm{~cm}$, single/double support time: 1.1/0.4 s).
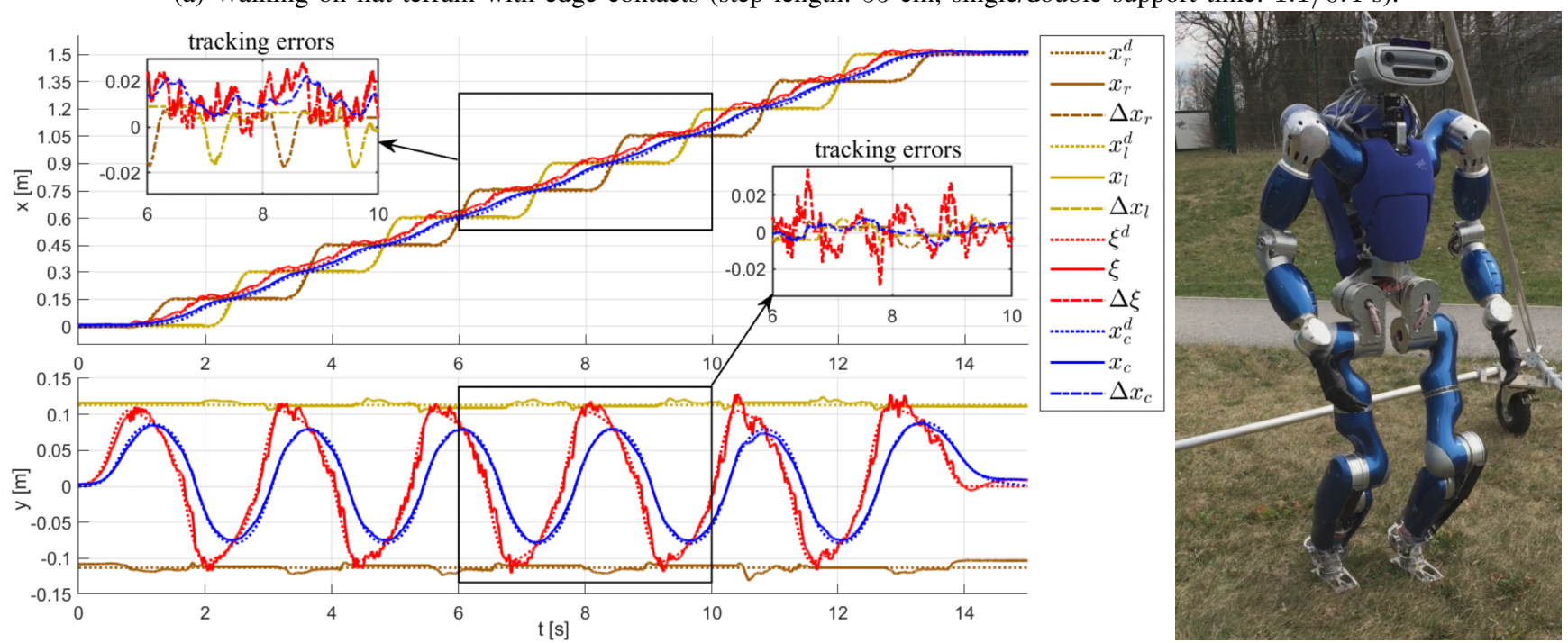

(b) Walking on grass (step length: $15 \mathrm{~cm}$, single/double support time: $0.9 / 0.3 \mathrm{~s}$, without stance foot stabilization).
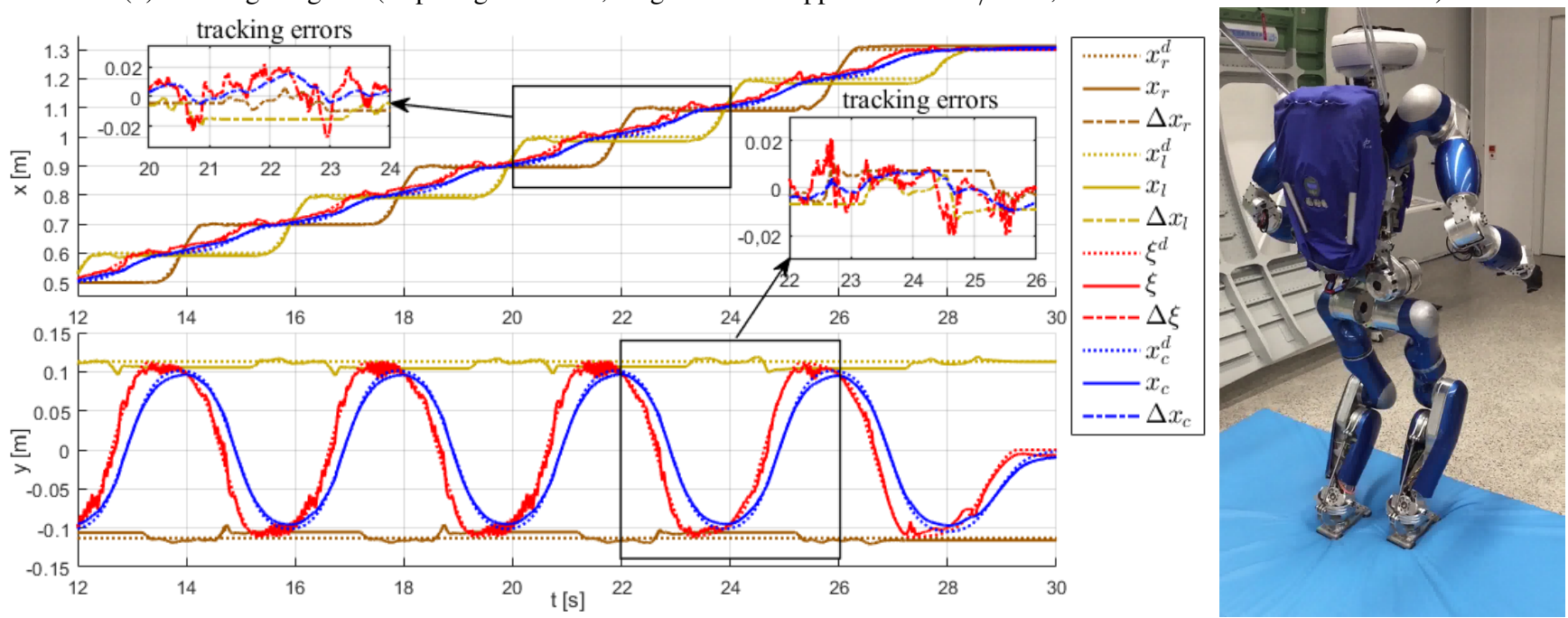

(c) Walking on mattress (step length: $10 \mathrm{~cm}$, single/double support time: $1.2 / 0.8 \mathrm{~s}$, with stance foot stabilization).

Fig. 5: Torque-based dynamic walking experiments with the humanoid robot TORO.

CoM tracking performance: the mean tracking error of the CoM is $6 \mathrm{~mm}$ on the $x$-axis (maximum error: $17 \mathrm{~mm}$ ) and 4 $\mathrm{mm}$ on the $y$-axis (maximum error: $10 \mathrm{~mm}$ ). We performed the second trial without stance foot stabilization, using the same walking parameters. A comparative video of the two experiments can be found in the multimedia attachment. 


\section{CONCLUSION AND FUTURE WORK}

In this paper, we presented a robust and effective method for dynamic walking with torque-controlled humanoid robots on compliant and uneven terrain. Our approach is based on the concepts of DCM and passivity-based whole-body control, which were discussed in detail in the corresponding sections. We introduced a generalized task formulation for the passivity-based controller, and implemented the dynamic walking controller in this framework. Additionally, we proposed a stabilization method for the stance foot while walking on compliant or rough terrain. Our proposed method achieves precise trajectory tracking and robust locomotion on various terrains, which we demonstrated with several experiments.

In our future research, we will compare the presented approach with the inverse dynamics based walking controller reported in [22] with respect to robustness, performance, and ease of use (parameter tuning, etc.). Additionally, we intend to integrate our DCM-based step adjustment [10] and an angular momentum task into the current framework.

\section{ACKNOWLEDGEMENT}

This project has received funding from the European Research Council (ERC) under the European Unions Horizon 2020 research and innovation programme (grant agreement No. 819358).

\section{REFERENCES}

[1] R. J. Full and D. E. Koditschek, "Templates and anchors: neuromechanical hypotheses of legged locomotion on land," Journal of Experimental Biology, vol. 202, no. 23, pp. 3325-3332, 1999.

[2] P.-B. Wieber, "Trajectory free linear model predictive control for stable walking in the presence of strong perturbations," in IEEE-RAS Int. Conf. Humanoid Robots, 2006, pp. 137-142.

[3] T. Koolen, S. Bertrand, G. Thomas, T. De Boer, T. Wu, J. Smith, J. Englsberger, and J. Pratt, "Design of a momentum-based control framework and application to the humanoid robot atlas," International Journal of Humanoid Robotics, vol. 13, no. 01, p. 1650007, 2016.

[4] P. M. Wensing and D. E. Orin, "High-speed humanoid running through control with a 3d-slip model," in IEEE-RAS Int. Conf. Humanoid Robots, 2013, pp. 5134-5140.

[5] J. Englsberger, P. Kozłowski, C. Ott, and A. Albu-Schäffer, "Biologically inspired deadbeat control for running: from human analysis to humanoid control and back," IEEE Trans. Robotics, vol. 32, no. 4, pp. 854-867, 2016.

[6] S. Kajita, F. Kanehiro, K. Kaneko, K. Yokoi, and H. Hirukawa, "The $3 \mathrm{~d}$ linear inverted pendulum mode: A simple modeling for a biped walking pattern generation," in IEEE/RSJ Int. Conf. Intelligent Robots and Systems, vol. 1, 2001, pp. 239-246.

[7] J. Englsberger, C. Ott, and A. Albu-Schäffer, "Three-dimensional bipedal walking control based on divergent component of motion," IEEE Trans. Robotics, vol. 31, no. 2, pp. 355-368, 2015.

[8] T. Takenaka, T. Matsumoto, and T. Yoshiike, "Real time motion generation and control for biped robot-1 st report: Walking gait pattern generation," in IEEE/RSJ Int. Conf. Intelligent Robots and Systems, 2009.

[9] J. Pratt, T. Koolen, T. De Boer, J. Rebula, S. Cotton, J. Carff, M. Johnson, and P. Neuhaus, "Capturability-based analysis and control of legged locomotion, part 2: Application to m2v2, a lower-body humanoid," Int. J. Robotics Research, vol. 31, no. 10, pp. 1117-1133, 2012.

[10] J. Englsberger, G. Mesesan, and C. Ott, "Smooth trajectory generation and push-recovery based on divergent component of motion," in IEEE/RSJ Int. Conf. Intelligent Robots and Systems, 2017, pp. 45604567.
[11] G. Mesesan, J. Englsberger, C. Ott, and A. Albu-Schäffer, "Convex properties of center-of-mass trajectories for locomotion based on divergent component of motion," IEEE Robot. Autom. Letters, vol. 3, no. 4, pp. 3449-3456, 2018.

[12] K. Nishiwaki and S. Kagami, "Walking control on uneven terrain with short cycle pattern generation," in IEEE-RAS Int. Conf. Humanoid Robots, 2007, pp. 447-453.

[13] M. Morisawa, F. Kanehiro, K. Kaneko, S. Kajita, and K. Yokoi, "Reactive biped walking control for a collision of a swinging foot on uneven terrain," in IEEE-RAS Int. Conf. Humanoid Robots, 2011, pp. 768-773.

[14] G. Cheng, S.-H. Hyon, J. Morimoto, A. Ude, J. G. Hale, G. Colvin, W. Scroggin, and S. C. Jacobsen, "CB: A humanoid research platform for exploring neuroscience," Advanced Robotics, vol. 21, no. 10, pp. 1097-1114, 2007.

[15] J. Englsberger, A. Werner, C. Ott, B. Henze, M. A. Roa, G. Garofalo, R. Burger, A. Beyer, O. Eiberger, K. Schmid, and A. Albu-Schäffer, "Overview of the torque-controlled humanoid robot TORO," in IEEERAS Int. Conf. Humanoid Robots, 2014, pp. 916-923.

[16] S.-H. Hyon, D. Suewaka, Y. Torii, and N. Oku, "Design and experimental evaluation of a fast torque-controlled hydraulic humanoid robot," IEEE/ASME Trans. Mechatronics, vol. 22, no. 2, pp. 623-634, 2016.

[17] B. Henze, A. Dietrich, M. A. Roa, and C. Ott, "Multi-contact balancing of humanoid robots in confined spaces: Utilizing knee contacts," in IEEE/RSJ Int. Conf. Intelligent Robots and Systems, 2017, pp. 697704.

[18] S. Feng, X. Xinjilefu, C. G. Atkeson, and J. Kim, "Optimization based controller design and implementation for the atlas robot in the darpa robotics challenge finals," in IEEE-RAS Int. Conf. Humanoid Robots, 2015, pp. 1028-1035.

[19] M. A. Hopkins, D. W. Hong, and A. Leonessa, "Compliant locomotion using whole-body control and divergent component of motion tracking," in IEEE Int. Conf. Robotics and Automation, 2015, pp. 57265733.

[20] B. J. Stephens and C. G. Atkeson, "Push recovery by stepping for humanoid robots with force controlled joints," in IEEE-RAS Int. Conf. Humanoid Robots, 2010, pp. 52-59.

[21] B. Henze, M. A. Roa, and C. Ott, "Passivity-based whole-body balancing for torque-controlled humanoid robots in multi-contact scenarios," Int. J. Robotics Research, vol. 35, no. 12, pp. 1522-1543, 2016.

[22] J. Englsberger, G. Mesesan, A. Werner, and C. Ott, "Torque-based dynamic walking - a long way from simulation to experiment," in IEEE Int. Conf. Robotics and Automation, 2018, pp. 440-447.

[23] S.-H. Hyon, J. G. Hale, and G. Cheng, "Full-body compliant humanhumanoid interaction: balancing in the presence of unknown external forces," IEEE Trans. Robotics, vol. 23, no. 5, pp. 884-898, 2007.

[24] B. Henze, A. Dietrich, and C. Ott, "An approach to combine balancing with hierarchical whole-body control for legged humanoid robots," IEEE Robot. Autom. Letters, vol. 1, no. 2, pp. 700-707, 2016.

[25] B. Paden and R. Panja, "Globally asymptotically stable 'pd+' controller for robot manipulators," International Journal of Control, vol. 47, no. 6, pp. 1697-1712, 1988.

[26] C. Ott, M. A. Roa, and G. Hirzinger, "Posture and balance control for biped robots based on contact force optimization," in IEEE-RAS Int. Conf. Humanoid Robots, 2011, pp. 26-33.

[27] M. Hosokawa, D. N. Nenchev, and T. Hamano, "The DCM generalized inverse: efficient body-wrench distribution in multi-contact balance control," Advanced Robotics, vol. 32, no. 14, pp. 778-792, 2018.

[28] G. Garofalo, B. Henze, J. Englsberger, and C. Ott, "On the inertially decoupled structure of the floating base robot dynamics," in Int. Conf. on Mathematical Modelling (MATHMOD), 2015, pp. 322-327.

[29] G. D. Niemeyer and J.-J. E. Slotine, "Performance in adaptive manipulator control," vol. 10, no. 2, pp. 149-161, 1991.

[30] H. J. Ferreau, H. G. Bock, and M. Diehl, "An online active set strategy to overcome the limitations of explicit MPC," International Journal of Robust and Nonlinear Control: IFAC-Affiliated Journal, vol. 18, no. 8, pp. 816-830, 2008.

[31] A. Albu-Schäffer, S. Haddadin, C. Ott, A. Stemmer, T. Wimböck, and G. Hirzinger, "The DLR lightweight robot: design and control concepts for robots in human environments," Industrial Robot: an international journal, vol. 34, no. 5, pp. 376-385, 2007. 